Department of Internal Medicine

Division of Gastroenterology (111D)

VA Medical Center/University of Michigan

Ann Arbor, Michigan 48105

Readers who wish to contribute to HEPATOLOGY Elsewhere are requested to write Dr. Jorge J. Gumucio, Editor, indicating topics they would like to comment on. They are welcome to telephone (313/769-7100, ext. 5796) to express their willingness to contribute, to indicate a specific article they think worthy of comment or to identify potential authors of future HEPATOLOGY Elsewhere commentaries.

These mini-editorials are intended to bring interesting articles to the attention of the readership and to put these papers into perspective. They may applaud or criticize, amplify, delimit or speculate about the significance of the paper.

The following instructions will apply: (a) The title of the review should prompt the reader's interest in the article to be reviewed. (b) Please include the names of all authors, the exact title of the article and the name of the journal followed by the exact reference. (c) Enclose a legible photocopy of the abstract. (d) The comments should be typed, double spaced and comprise approximately four to five manuscript pages. (e) In addition to the comments, include a short reference list (up to 10 references) containing the names of all authors, using the HEPATOLOGY format (see "Instructions to Authors"). (f) At the close of the comments, include the reviewers' complete names and affiliations. (g) Send the review to the address listed above.

The authors of the original paper will not be requested to prepare a response to be published along with the review. The authors and others, however, have the opportunity to respond in the Correspondence section of HEPATOLOGY Elsewhere, and these comments, along with a short response by the editorialist, will be published as promptly as possible.

The authors' comments must conform to the following style: (a) A high level of professional discussion has to be maintained. Comments that the section editor considers offensive to either the author (in the original review) or to the reviewer (in the author's response) will not be published. (b) The comments should not exceed two double-spaced manuscript pages and five references.

The reviewer has the opportunity of responding to the author's comments using an equal format. Elsewhere will not publish subsequent letters dealing with the same discussion.

Note: Abstracts are reproduced with the permission of the authors and publishers exactly as they appear in the original articles. When an abstract is not published as part of the article under comment or when permission to reproduce the abstract is not forthcoming, an "Editor's Abstract" is prepared instead.

\section{Elsewhere Reviews}

\section{PREDICTING POSTPROCEDURE BLEEDING IN LIVER DISEASE}

McVay PA, Toy PTCY. Lack of increased bleeding after liver biopsy in patients with mild hemostatic abnormalities. Am J Clin Pathol 1990;94:747-753.

\section{ABSTRACT}

Prophylactic transfusions of fresh frozen plasma and platelets are sometimes given to patients with mild elevations in prothrombin time (PT) and partial thromboplastin time (PTT) and mild thrombocytopenia before percutaneous liver biopsy. To determine whether PTs and PTTs 1.1-1.5 times midrange normal levels and platelet counts $50.99 \times 10^{9} / \mathrm{L}$ are associated with increased bleeding complications, hospital records of all patients who underwent percutaneous liver biopsy during 56 consecutive months $(n=291)$ were reviewed. Complete information was available for 177 inpatient procedures (155 standard, 22 fine needle). Overall, the frequency of bleeding complications in patients with platelet counts $\geq 50 \times 10^{9} / \mathrm{L}$ was $3.4 \%$ ( 6 of 175), with no significant difference between patients with mild hemostatic abnormalities and patients with normal parameters. These data suggest that prophylactic transfusions may not be necessary. One factor was highly associated with bleeding complications: a patient diagnosis of malignancy, $14 \%$ ( 7 of 50 ) compared with $0.8 \%$ ( 1 of 127) among other patients $(P<0.001)$. These patients should be monitored closely after biopsy.

McVay PA, Toy PTCY. Lack of increased bleeding after paracentesis and thoracentesis in patients with mild coagulation abnormalities. Transfusion 1991;31:164-171.

\section{ABSTRACT}

To determine whether untreated mild coagulopathy in patients with no evidence of clinical bleeding is associated with an increased risk of hemorrhage after paracentesis or thoracentesis, retrospective examination was conducted of 608 consecutive procedures for which prothrombin time (PT), partial thromboplastin time (PTT), platelet (Plt) counts, and preprocedure and postprocedure hemoglobin concentrations were available. There was no increased bleeding in patients with mild to moderate coagulopathy (defined as PT or PTT up to twice the midpoint normal range or plt count of 50 to $99 \times 10^{3}$ per $\left.\mu \mathrm{L}\left[50-99 \times 10^{8} / \mathrm{L}\right]\right)$. However, patients with markedly elevated serum creatinine levels $(6.0$ to $14.0 \mathrm{mg} / \mathrm{dL}$ [530-1240 $\mu \mathrm{mol} / \mathrm{L}])$ had a significantly greater average hemoglobin loss $(-0.82 \pm 1.3 \mathrm{~g} / \mathrm{dL}[-8 \pm 13 \mathrm{~g} / \mathrm{L}], \mathrm{n}=11)$ than patients 
with normal serum creatinine levels $(-0.12 \pm 0.88 \mathrm{~g} / \mathrm{dL}$ $[-1 \pm 9 \mathrm{~g} / \mathrm{L}], n=450)(p=0.011)$. Overall, the frequency of bleeding complications requiring red cell transfusions was very low: 0.2 percent of events. The most common diagnosis for patients who had paracentesis was alcoholic liver disease (72\%); for those having thoracentesis, it was infection (37\%). It can be concluded that, for these patients, prophylactic plasma or platelet transfusions are not necessary. Patients with markedly elevated serum creatinine deserve close postprocedure observation.

\section{COMMENTS}

It is almost a truism that patients with liver disease bleed, and may bleed severely. This leads to some trepidation when one is faced with performing an invasive procedure such as liver biopsy on a patient with liver disease and coagulopathy. Prophylactic plasma or platelet transfusions are often ordered before invasive procedures are performed in such patients, although scant evidence of their effectiveness exists. Moreover, increased awareness of the risks of blood transfusion has prompted greater conservatism in their use. Thus a need exists for the development of guidelines related to specific clinical situations, including correction of coagulopathies before procedures. Recently, McVay and Toy published two studies regarding the risk of bleeding in patients with mild coagulopathies undergoing invasive procedures, with an eye to determining the value of prophylactic plasma or platelet transfusion in this setting.

The first study was a retrospective examination of patients at the San Francisco General Hospital undergoing percutaneous liver biopsies in a 56-mo period. Patients who received prophylactic fresh frozen plasma or who had active bleeding unrelated to the procedure were excluded. A bleeding complication was defined as a hemoglobin decrease of $20 \mathrm{gm} / \mathrm{L}$, or more after the biopsy, allowing for any transfused blood. In addition, clinical evidence of bleeding was sought. Most biopsies were performed with Menghini-style Klatskin needles, with Tru-Cut and fine needles used occasionally. To define the degree of coagulopathy, the measured prothrombin time (PT) and partial thromboplastin time (PTT) were compared with the midpoint and the normal range for their laboratory. By the authors' convention, mild coagulopathy was considered to be PT up to 1.5 times control or PTT up to 2.0 times control. Normal platelet counts were considered greater than $100 \times 10^{9} / \mathrm{L}$, with mild thrombocytopenia in the range 50 to $99 \times 10^{9} / \mathrm{L}$ and moderate-to-marked thrombocytopenia as counts below that.

These authors identified 177 eligible patients, who were stratified according to degree of coagulopathy. The overall frequency of bleeding complications was $4.5 \%$ of the patients, with $3.4 \%$ of the patients requiring $\mathrm{RBC}$ transfusions. No statistically significant difference in bleeding complications was found between patients with normal PT or PTT and those with mildly elevated levels. In addition, no difference was found between patients with normal platelet counts and those with mild thrombocytopenia. Two of 14 patients with moderately pro- longed PTT and both patients with moderate-to-marked thrombocytopenia bled, but too few patients were included in these groups to reach a meaningful conclusion. One factor that was significantly associated with postbiopsy bleeding was a diagnosis of malignancy. However, because a biopsy is most often performed in such patients to establish the presence of malignancy, this information usually is not available before the procedure.

The second study, which was of similar design, was of patients undergoing paracentesis or thoracentesis in a 30 -mo time period. A total of 608 procedures involved 395 patients. Of these patients, $51 \%$ had alcoholic liver disease (the single largest category) or hepatitis. The overall rate of bleeding complications in a cohort of patients with coagulopathy comparable with those in the foregoing study was $3 \%$, and only once was RBC transfusion required. Again, the authors found no significant difference in bleeding complications between patients with normal and those with mild to moderately prolonged PT or PTT. Mild-to-moderate thrombocytopenia was also not predictive of bleeding; however, kidney failure with creatinine levels greater than 530 $\mu \mathrm{mol} / \mathrm{L}(6.0 \mathrm{mg} / \mathrm{dl})$ was significantly associated with postbiopsy bleeding, most likely caused by acquired platelet dysfunction. In this article, the authors separately considered patients who received prophylactic fresh frozen plasma transfusions. Although the numbers were small ( 43 procedures in 35 patients), a greater proportion of patients with more severe coagulopathies was seen; however, the incidence of bleeding complications in these patients did not differ from those in those patients who did not have transfusions.

Both these studies may be criticized as being retrospective and involving a relatively small number of patients; however, the logistic considerations in performing a large randomized prospective trial are daunting. The authors calculated that to detect a doubling of the complication rate in liver biopsies would require 950 patients. Such a study is unlikely to be undertaken. The rate of bleeding after liver biopsy was higher than reported by others, but this rate may be accounted for by the selection of carefully monitored inpatients who might be expected to have higher rates of complications and by the relatively liberal definitions of bleeding based on hemoglobin concentrations.

If we cannot use the common indices of coagulation to predict bleeding in patients undergoing these invasive procedures, can the bleeding time be used? The bleeding time is a test with great intuitive appeal. The name suggests that it will tell you how long a patient will bleed. Unfortunately, the predictive value of the bleeding time is open to serious question. In a recent comprehensive review, Lind (1) notes that although little evidence exists to support using the bleeding time as a predictive indicator, it continues to be used as a screening test because of medical custom and because it has been recommended for many years. In addition we make assumptions about the test that may not be warranted. These assumptions imply that the skin bleeding time 
correlates with bleeding at other sites, that it is diagnostic and prognostic in von Willebrand's disease, that drugs altering platelet function have a consistent effect on the bleeding time and that "surgical" bleeding, such as that caused by biopsy, is synonymous with "clinical" bleeding. Specifically, with respect to liver biopsy Lind found only one paper addressing the bleeding time in this situation.

Blake and colleagues (2) studied 100 consecutive patients with cirrhosis, 42 of whom had prolonged bleeding times, which was defined as more than $10 \mathrm{~min}$ by the Simplate II method. Although not specifically stated, all patients apparently underwent liver biopsy, most during a previous admission. Complications of biopsy are not discussed, but because the bleeding time was measured at least 10 days after any bleeding episode, it seems unlikely that any serious postbiopsy bleeding occurred. They found that of the parameters they measured only serum bilirubin and platelet count were independently correlated with bleeding time. Fourteen patients had prolonged bleeding times despite normal platelet counts. These authors, citing other authorities, consider a platelet count of greater than $80 \times 10^{9} / \mathrm{L}$ and PT less than 17 sec to be "safe limits" for performing percutaneous liver biopsy, criteria that seem overly conservative in light of the studies of McVay and Toy. The authors also recommend that "before having invasive procedures patients with raised bilirubin concentrations or poor hepatic function should have their bleeding time measured," but they do not suggest how this information is to be used in planning the procedure. Indeed, from their data, if a normal bleeding time and their "safe limits" are used as selection criteria, only $29 \%$ of patients with cirrhosis may have biopsies without risk of hemorrhage. Although it is interesting that many cirrhotic patients have an unexpected prolonged bleeding time, it clearly is of little value in assessing the need for prophylactic transfusion before invasive procedures.

When should one administer prophylactic blood products to patients with liver disease? The jury is still out; however, it appears that even though we have no reliable means of predicting who will bleed after an invasive procedure, mild-to-moderate coagulopathy seems to pose little, if any, increased risk of bleeding. Therefore we may conclude the prophylactic plasma or platelet transfusions, with their attendant hazards, are not indicated in this setting.

ROBERTSON D. DAVENPORT, M.D. Blood Bank and Transfusion Service The University of Michigan Hospitals Ann Arbor, Michigan 48109-0054

\section{REFERENCES}

1. Lind SE. The bleeding time does not predict surgical bleeding. Blood 1991;77:2547-2552.

2. Blake JC, Sprengers D, Grech P, McCormick PA, McIntyre N Burroughs AK. Bleeding time in patients with hepatic cirrhosis. BMJ 1990;301:12-15.

\section{CHOLESCINTIGRAPHY IN ACALCULOUS BILIARY}

\section{PAIN: IF ABNORMAL, SHOULD} CHOLECYSTECTOMY FOLLOW?

Yap L, Wycherley AG, Morphett AD, Toouli J. Acalculous biliary pain: cholecystectomy alleviates symptoms in patients with abnormal cholescintigraphy. Gastroenterology 1991;101:786-793.

\section{ABSTRACT}

A 45-minute infusion of an octapeptide of cholecystokinin (Kinevac; Squibb Diagnostics, New Brunswick, NJ) was used to measure the gallbladder ejection fraction during cholescintigraphy in 40 normal volunteers. Cholecystokinin cholescintigraphy was shown to be a reproducible test. The maximum mean gallbladder ejection fraction occurred 15 minutes after cholecystokinin infusion and was $74.5 \% \pm 1.9 \%$ (mean \pm SEM). A gallbladder ejection fraction $>40 \%$ (mean - 3SD) was arbitrarily defined to be normal. The gallbladder ejection fraction test was then used to identify patients with acalculous biliary symptoms who may respond to cholecystectomy. A total of 103 patients was tested; 21 had abnormal gallbladder ejection fractions and were randomized into two groups, cholecystectomy or no operation. These patients were followed up symptomatically at 3-month intervals for 13-54 months (mean, 34 months). Of the 11 patients who underwent cholecystectomy, $10(91 \%)$ lost their symptoms and 1 improved. Of the 10 patients in the group that did not undergo surgery, all continued to be symptomatic, 2 of whom requested cholecystectomy after 13 and 24 months, respectively. Of the 13 gallbladders obtained from surgery, 12 showed evidence of chronic cholecystitis, muscle hypertrophy, and/or narrowed cystic duct. A normal gallbladder ejection fraction was recorded in 82 patients, and further treatment was left to the discretion of their referring clinician. On follow-up, 50 patients were asymptomatic and 10 were symptomatic without specific treatment of the biliary tract; 14 underwent cholecystectomy, 8 of whom were asymptomatic. Pathological abnormalities were recorded in 6 of the removed gallbladders. It is concluded that the gallbladder ejection fraction obtained after a 45minute infusion of cholecystokinin during cholescintigraphy is a reproducible measure of gallbladder emptying, and that cholecystectomy alleviates the biliary-type pain of patients with a reduced gallbladder ejection fraction.

\section{COMMENTS}

Chronic right upper quadrant pain without gallstones, usually identified as "acalculous biliary pain," has been clinically difficult to define in the absence of gallstones. The advent of more simplified surgery in the form of laparoscopic cholecystectomy presents, perhaps, too easy an alternative to a challenging question: what test will detect subtle gallbladder-biliary tract disease and predict a favorable outcome from surgery?

The prospective study by Yap et al. would indicate that cholecystokinin (CCK) cholescintigraphy is the answer. Using a standard infusion of CCK-8 $(9 \mathrm{ng} / \mathrm{kg} / \mathrm{hr})$ for 45 min in 108 patients considered to have biliary-type pain, 21 were found to have a gallbladder ejection fraction less 